\title{
Identification of 217 unreported mutations in the $F 8$ gene in a group of 1,410 unselected Italian patients with hemophilia $A$
}

\author{
R. Santacroce $\cdot$ M. Acquila $\cdot$ D. Belvini $\cdot$ G. Castaldo $\cdot$ I. Garagiola $\cdot$ \\ S. H. Giacomelli $\cdot$ A. M. Lombardi - B. Minuti - F. Riccardi $\cdot$ R. Salviato \\ L. Tagliabue - E. Grandone - M. Margaglione $\cdot$ The AICE-Genetics Study Group
}

Received: 5 November 2007/Accepted: 26 November 2007/Published online: 23 January 2008

(C) The Japan Society of Human Genetics and Springer 2008

\begin{abstract}
To provide a National database, 1,410 unrelated hemophilia A (HA) patients were investigated using screening methods denaturing high-performance liquid chromatography (DHPLC), conformational-sensitive gel electrophoresis (CSGE)] and/or direct sequencing. $F 8$ gene mutations were identified in $877(81 \%), 146(82 \%)$, and $133(89 \%)$ families with severe, moderate, or mild HA, respectively. Among the 382 different mutations detected, 217 (57\%) have not previously been reported in the F8 Haemophilia A Mutation, Structure, Test and Resource Site (HAMSTeRS) database. Mutations leading to a null allele accounted for $82,15 \%$, and less than $1 \%$ of severe, moderate, or mild HA, respectively. A missense mutation was identified in $16 \%, 68 \%$, and $81 \%$ of severe, moderate,
\end{abstract}

R. Santacroce $\cdot$ M. Margaglione $(\square)$

Cattedra di Genetica Medica, Dipartimento di Scienze

Biomediche, Università degli Studi di Foggia,

viale Pinto, Foggia 71100, Italy

e-mail:m.margaglione@unifg.it

M. Acquila

Laboratorio di Ematologia ed Emofilia, IV Divisione

di Pediatria, Istituto Gaslini, Genova, Italy

D. Belvini $\cdot$ R. Salviato

Servizio Trasfusionale, Castelfranco Veneto Hospital (TV),

ASL 8 Regione Veneto, Veneto, Italy

G. Castaldo

Dipartimento di Biochimica e Biotecnologie Mediche, Università di Napoli "Federico II", CEINGE Biotecnologie

Avanzate, Napoli, Italy

I. Garagiola $\cdot$ L. Tagliabue

"Angelo Bianchi Bonomi" Hemophilia and Thrombosis Center,

University of Milan and IRCCS Maggiore Hospital,

Mangiagalli and regina Elena Foundation, Milan, Italy or mild HA, respectively. They included 105 missense mutations (48\%), 41 small deletions (19\%), 25 splice site mutations (12\%), 24 nonsense mutations (11\%), 18 insertions $(8 \%)$, three large deletions $(1 \%)$, and one deletion plus insertion. Unreported mutations were distributed throughout the $F 8$ gene, as they affected all $F 8$ exons but exon 20. We report a wide spectrum of mutations collected in a large National database. The type of mutation was a strong predictor of the clinical phenotype. This database is expected to considerably improve the genetic counseling and medical care of HA families in Italy.

Keywords Hemophilia A · F8 gene - Mutations · Phenotype

S. H. Giacomelli

Divisione di Ematologia, "S. Bortolo" Hospital,

Vicenza, Italy

A. M. Lombardi

Dipartimento di Scienze Mediche e Chirurgiche,

Università di Padova, Padova, Italy

B. Minuti

SOD Diagnostica Genetica, Azienda Ospedaliero-Universitaria

Careggi Firenze, Firenze, Italy

F. Riccardi

UOS di Genetica Molecolare e Citogenetica, Centro di

Riferimento Regionale per l'Emofilia e le Malattie Emorragiche

Congenite, University Hospital of Parma, Parma, Italy

E. Grandone - M. Margaglione

Unita' di Emostasi e Trombosi, I.R.C.C.S. "Casa Sollievo

della Sofferenza", S. Giovanni Rotondo, Italy 


\section{Introduction}

Hemophilia A (HA) is an X-linked bleeding disorder caused by heterogeneous mutations in the Factor 8 (F8) gene. Its incidence is estimated at 1:5,000-10,000 in men (Kazazian et al. 1995). The $F 8$ gene maps to the distal end of the long arm of X-chromosome (Xq28) and spans $186 \mathrm{~kb}$ of genomic DNA. It consists of 26 exons and encodes a mature protein of 2,332 amino acids (Kazazian et al. 1995). Over the last decades, rapidly increasing numbers of causative gene alterations have been described in different ethnic groups. Actually, more than 900 mutations within the $F 8$ coding and untranslated regions have been identified and listed in the F8 Haemophilia A Mutation, Structure, Test and Resource Site (HAMSTeRS) mutation database (URL: http://europium.csc.mrc.ac.uk) (Gitschier et al. 1984). These findings further support the concept that HA has a very high mutational heterogeneity and represents a challenge for the provision of genetic services because carrier and prenatal diagnosis cannot be based on the screening of a limited number of common mutations (Kemball-Cook et al. 1998). The exponential discovery rate of new genomic alterations, leading to HA, as well as the need for comparative studies of different population's mutation frequencies, requires recording their population-wide spectrum in mutation databases. These databases are likely to facilitate genetic consultation of patients' families and can help optimize national DNA diagnostic services also by enhancing awareness among clinicians, patients, and the general public. Finally, mutation analysis of a given population is useful for further understanding structural and functional aspects of the mutant protein and the correlation between genotype and phenotype. Therefore, the Italian Association of Hemophilia Centres (AICE) planned to set up a National database aiming at characterizing the $F 8$ gene mutations to elucidate molecular basis of HA in Italy and to provide insight into protein structure-function relationships, unraveling the correlation (if any) between genotype and clinical phenotype.

In this study, we report phenotypic and genotypic data from 1,410 independent HA families comprising about $82 \%$ of Italian HA patients. These data strengthen the relevance for a National Hemophilia Registry that is expected to considerably improve the medical care of HA families in Italy with respect to treatment and genetic counseling.

\section{Materials and methods}

The study was carried out according to the Principles of the Declaration of Helsinki; informed consent was obtained from all participants. All patients involved in this study are regularly followed at one of 49 hemophilia treatment centers (see "Appendix") spread all over the country and belonging to AICE (URL: http://www.aiceonline.it), an organization founded with the aim of organizing national clinical and research activities in inherited bleeding disorders. Patients were considered to have HA according to the international consensus of the 2001 International Society on Thrombosis and Haemostasis (ISTH) Factor VIII and Factor IX Subcommittee (White et al. 2001). All known HA patients are registered and followed at one of these centers. For each patient, clinical and laboratory data (including FVIII clotting activity and inhibitor) are recorded.

\section{DNA collection}

A blood sample $(5-10 \mathrm{~mL})$ was collected in ethylenediaminetetraacetate (EDTA) or sodium citrate from each patient and stored at $-20^{\circ} \mathrm{C}$ until sent to one of the nine laboratories that performed the genetic analysis. Isolation of DNA from leukocytes was carried out according to standard procedures (Miller et al. 1988).

\section{PCR detection of the introns 1 and 22 inversion}

Intron 1 inversions were detected by polymerase chain reaction (PCR), as reported earlier (Bagnall et al. 2002). Long PCR for detecting the intron 22 inversion was performed as previously described (Liu et al. 1998).

\section{Amplification of the $F 8$ gene}

PCR was carried out according to standard procedures (D'Andrea et al. 2002). A total of $14 \mathrm{~kb}$ of the $F 8$ gene, including the entire coding sequence, exon-intron junctions, and part of the $5^{\prime}$ and $3^{\prime}$ untranslated regions, were amplified by PCR. Most of the primers and amplification conditions have been previously reported; primer sequences, annealing temperatures, and the size of PCR fragments are available on request.

\section{Mutation detection screening}

Mutational analysis was performed through all of the coding exons and the exon/intron boundaries of the $F 8$ gene using denaturing high-performance liquid chromatography (DHPLC) analysis in four of the nine laboratories (Firenze, Genova, Parma, and Vicenza), whereas another 
one (Castelfranco Veneto) used conformational-sensitive gel electrophoresis (CSGE). The success rate ranged from $80 \%$ to $95 \%$. All amplification products that showed an abnormal pattern were then analyzed by direct sequencing. The remaining four laboratories (Foggia, Milano, Napoli, and Padova) did not employ any screening method, and samples were subjected to direct gene sequencing.

\section{DNA sequencing}

Amplified DNA fragments were purified and subjected to direct cycle sequence analysis using the Taq dye-deoxy terminator method and an ABI PRISM 3100 genetic Analyzer sequencer (Applied Biosystems, Foster City, CA, USA) according to the manufacturer's instructions. All the nucleotide changes identified were confirmed by repeating the PCR and sequencing reactions. To address whether new mutations identified would be a polymorphism, 100 control subjects from the same ethnic background were investigated. Mutation nomenclature was according to the F8 HAMSTeRS mutation database (URL: http://europium. csc.mrc.ac.uk).

\section{Splice-site prediction}

Missense mutations and mutations at or near the splice junction consensus sequences were analyzed by the Splice Site Prediction program (http://www.fruitfly.org/seq_tools/ splice.html) to predict the changes in RNA splicing.

\section{Results}

F8 gene mutations were identified in 1,156 out of 1,410 unrelated families with a history of HA, representing a mutation detection rate of $82 \%$. Among these unrelated families, at least one causal $F 8$ mutation was identified in $877(81 \%), 146(82 \%)$, and $133(89 \%)$ families with severe, moderate, or mild HA, respectively. Among severe HA patients, in addition to intron 22 and 1 inversion, 272 different mutations were recorded, including 100 missense mutations, 63 small deletions, 47 nonsense mutations, 25 splice-site mutations, 24 small insertions, 11 large deletions (seven spanning more than one domain), and two involving different mechanisms (deletion + insertion; missense/splicing) (Fig. 1a). In patients presenting with moderate HA, 83 different mutations were recorded, including 59 missense mutations, six splice-site mutations, six small deletions, six nonsense mutations, five small insertion, and one involving a different mechanism (duplication of exon 13) (Acquila et al. 2004) (Fig. 1b).

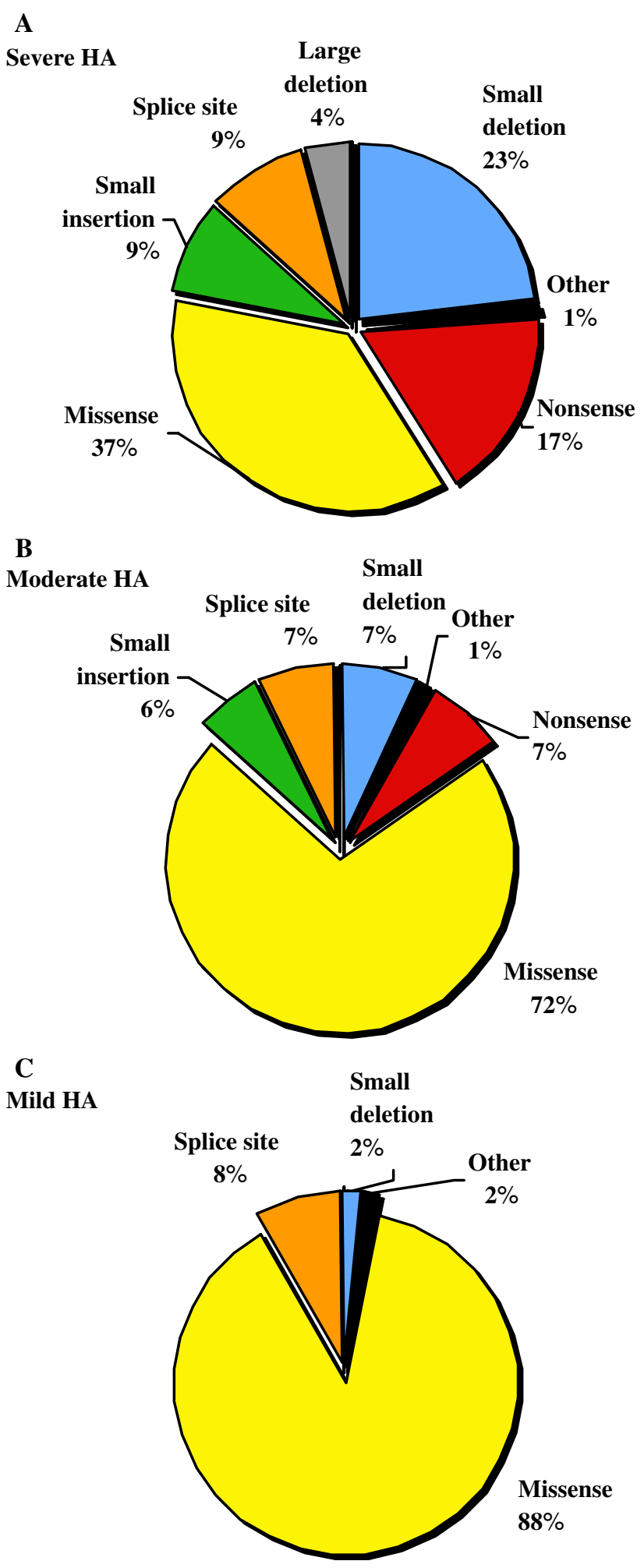

Fig. 1 Distribution of the different $F 8$ mutation types in unrelated hemophilia (HA) families according to the clinical phenotype. a Severe HA, b moderate HA, c mild HA

Finally, in mild HA patients, 57 different mutations were found, including 50 missense mutations, five splice-site mutations, one small deletion, and duplication of exon 13 
(Acquila et al. 2004) (Fig. 1c). Overall, mutations that are likely to give rise to a null allele (inversions of an intron, deletions, insertions, and nonsense mutations) accounted for $82 \%$ of severe, $15 \%$ of moderate, and less than $1 \%$ of mild HA families. On the other hand, a missense mutation was identified in $16 \%$ of severe, $68 \%$ of moderate, and $81 \%$ of mild HA families. Mutations predicted to affect $F 8$ mRNA splicing occurred in $4 \%$ of severe, $16 \%$ of moderate, and $5 \%$ of mild HA families, suggesting that for most of splice-site mutations, by escaping aberrant splicing, a rescue of low levels of normal transcripts may take place.

Among the 384 different mutations detected, 217 (57\%) have not previously been reported in the F8 HAMSTeRS mutation database. They include 105 missense mutations (48\%), 41 small deletions (19\%), 25 splice-site mutations (12\%), 24 nonsense mutations (11\%), 18 insertions (8\%), three large deletions $(1 \%)$, and one deletion + insertion (Table 1). Unreported mutations were distributed throughout the $F 8$ gene, as they affected all $F 8$ exons but exon 20.

Exon 14 accounts for approximately 43\% (3,106 out of $7,227 \mathrm{bp}$ ) of the coding region. Unreported mutations were found more frequently in exon 14, with 47 of 233 different mutations $(21 \%)$ occurring in this portion of the $F 8$ gene. Novel mutations identified consisted mainly in premature terminal codon-causing mutations with 19 small deletions (41\%), 11 insertions (23\%), 11 nonsense mutations (23\%), two splice-site mutations (4\%), and only four missense mutations $(9 \%)$.

\section{Discussion}

We report herein the largest series of $F 8$ analysis in HA patients issued in Italy. The vast majority of Italian families with HA, especially those with the severe cases, were included in this study, and it may be considered to be representative for the purpose of a population-based study of mutational heterogeneity. Actually, we were able to find 384 different mutations among 1,410 unrelated families. In accordance with previous data, more than $25 \%$ of these unrelated families of our cohort presented a novel mutation (Ljung and Sjorin 1999; Habart et al. 2003; Oldenburg and Pavlova 2006). This reflects the high degree of heterogeneity of the $F 8$-affecting mutations outside inversion of introns 22 and 1 . Our study confirms the well-known correlation between the type of mutation and the severity of HA. The type of mutations found was in agreement with results reported in other settings (Oldenburg and Pavlova 2006; Fernández-López et al. 2005; Jayandharan et al. 2005; Deszo et al. 2006). As would be expected, most severe HA patients carry a molecular defect leading to a null allele (large deletion, inversion, nonsense, and insertion/deletion mutations), whereas missense mutations have been found in the majority of moderate $(68 \%)$ and mild HA patients $(81 \%)$. Likewise, most mutations predicted to affect $F 8$ mRNA splicing occurred in moderate or mild HA families, suggesting that low levels of normal transcripts may escape aberrant splicing. To improve genetic counseling and medical care of HA families, mutations identified in this work will be available at the HAMSTeRS mutation database.

Exon 14 represents about one half of the coding region and encodes for the $F 8 \mathrm{~B}$ domain, a region lacking in procoagulant activity that is spliced out from the mature protein. This finding explains the occurrence of very few missense mutations in the central portion of exon 14 in our series and in other settings (see: HAMSTeRS mutation database).

In conclusion, we report a large National database in which a wide spectrum of new mutations has been collected. The type of mutation was a strong predictor of the clinical phenotype. This database will be of great value for genetic counseling in HA and is expected to considerably improve the medical care of HA families in Italy.

Acknowledgments Our sincere thanks to Prof. G. Mori for his efforts to set up the Italian HA mutation database. Without his help, this work would never have been accomplished.

\section{Appendix}

Alessandria: Dr.ssa L. Contino; Arezzo: Dr.ssa A. Accorsi; Bari "Policlinico" I: Dr. A. Scaraggi; Bari "Policlinico" II: Dr. N. Ciavarella; Bologna: Dr.ssa G. Rodorigo; Cagliari: Dr. R. Targhetta; Castelfranco Veneto: Dr. G. Tagariello, Dr.ssa D. Belvini, Dr.ssa R. Salviato; Catania: Dr. R. Musso; Catanzaro: Dr. G. Muleo; Cesena: Dr.ssa C. Biasoli; Cosenza: Dr.ssa V. Rossi; Cremona: Dr.ssa S. Testa; Ferrara: Dr. GL. Scapoli; Faenza: Dr. D. Vincenti; Firenze: Dr. M. Morfini; Genova: Dr. AC. Molinari, Dr.ssa MP. Bicocchi; Ivrea: Dr. M. Girotto; L'Aquila: Prof. G. Mariani; Latina: Dr. C. Ciabatta; Macerata: Dr.ssa MT. Carloni; Milano Policlinico: Prof. PM. Mannucci: Dr.ssa E. Santagostino: Prof.ssa F. Peyvandi; Milano Niguarda: Dr. F. Baudo; Modena: Dr. M. Marietta; Napoli "Ospedale Pediatrico Pausillipon": Dr. C. Perricone, Dr. M. Schiavulli; Napoli "Ospedale San Giovanni Bosco": Dr.ssa A. Rocino; Napoli "Policlinico Universitario Federico II": Prof. G. Di Minno, Dr. A. Coppola; Orvieto: Dr. M. Berrettini; Padova: Dr. E. Zanon; Palermo "Ospedale G. di Cristina": Prof. G. Mancuso; Palermo "Policlinico Universitario": Prof. S. Siragusa; Parma: Dr.ssa A. Tagliaferri: Dr.ssa F. Rivolta; Pavia: Prof.ssa G. Gamba; Perugia: Dr. 
Table 1 F8 mutations identified unreported in the Haemophilia A Mutation, Structure, Test and Resource Site (HAMSTeRS) mutation database

\begin{tabular}{|c|c|c|c|c|c|}
\hline Location & Nucleotide & Protein & Type & Effect & $\begin{array}{l}\text { Clinical } \\
\text { phenotype }\end{array}$ \\
\hline Exon 1 & c. $-43 \mathrm{C}>\mathrm{T}$ & & substitution & Transcription & Mild \\
\hline Exon 1 & c. $-25 \mathrm{~A}>\mathrm{G}$ & & Substitution & Transcription & Severe \\
\hline Exon 1 & c. $38 \mathrm{~T}>\mathrm{G}$ & p.Leu-7Arg & Substitution & Missense & Severe \\
\hline Exon 1 & c. $63 \mathrm{~A}>\mathrm{G}$ & p.Arg3Gly & Substitution & Missense & Severe \\
\hline Exon 1 & c.72_188 del15bp & p.Tyr5_Val10del & Deletion & Small deletion & Moderate \\
\hline Exon 1 & c. $102 \mathrm{C}>\mathrm{G}$ & p.Asp15Glu & Substitution & Missense & Mild \\
\hline Exon 1 & c. $104 \mathrm{~A}>\mathrm{G}$ & p.Tyr16Cys & Substitution & Missense & Severe \\
\hline Intron 2 & c. $144-3 \mathrm{C}>\mathrm{G}$ & & Substitution & Splicing & Severe \\
\hline Intron 2 & c. $144-2 \mathrm{~A}>\mathrm{T}$ & & Substitution & Splicing & Severe \\
\hline Exons 2-6 & & & Deletion & Large deletion & Severe \\
\hline Exon 2 & c.202_203 delCT & p.Thr49del & Deletion & Small deletion & Severe \\
\hline Exon 2 & c. $214 \mathrm{G}>\mathrm{C}$ & p.Glu53Asp & Substitution & Missense & Mild \\
\hline Exon 3 & c. $275 \mathrm{G}>\mathrm{A}$ & p.Gly73Asp & Substitution & Missense & Severe \\
\hline Exon 3 & c. $276 \mathrm{C}>\mathrm{T}$ & p.Pro74Ser & Substitution & Missense & Moderate \\
\hline Exon 3 & c.288_293 del GGCTGA & p.Gln77_Ala78del & Deletion & Small deletion & Severe \\
\hline Exon 3 & c. $308 \mathrm{~T}>\mathrm{A}$ & p.Val84Glu & Substitution & Missense & Severe \\
\hline Exon 3 & c. $339 \mathrm{~T}>\mathrm{A}$ & p.His94Gln & Substitution & Missense & Severe \\
\hline Exon 3 & c. $341 \mathrm{C}>\mathrm{T}$ & p.Pro95Leu & Substitution & Missense & Moderate \\
\hline Exon 3 & c. $343 \mathrm{~T}>\mathrm{G}$ & p.Val96Gly & Substitution & Missense & Moderate \\
\hline Exon 3 & c.347_353 delGTCTTCA & p.Ser97_His99del & Deletion & Small deletion & Severe \\
\hline Exon 3 & c. $350 \mathrm{~T}>\mathrm{C}$ & p.Leu98Pro & Substitution & Missense & Severe \\
\hline Exon 3 & c. $353 \mathrm{~A}>\mathrm{G}$ & p.His99Arg & Substitution & Missense & Moderate \\
\hline Exon 2 & c. $360 \mathrm{G}>\mathrm{T}$ & p.Gly102Val & Substitution & Missense & Moderate \\
\hline Exon 3 & c. $362 \mathrm{G}>\mathrm{C}$ & p.Gly102Ala & Substitution & Missense & Severe \\
\hline Exon 3 & c. $368 \mathrm{C}>\mathrm{G}$ & p. Ser104Stop & Substitution & Nonsense & Severe \\
\hline Exon 3 & c. $392 \mathrm{~A}>\mathrm{G}$ & p.Ile76Val & Substitution & Missense & Mild \\
\hline Intron 3 & c. $388+5 \mathrm{G}>\mathrm{A}$ & & Substitution & Splicing & Severe \\
\hline Exon 4 & c. $403 \mathrm{G}>\mathrm{T}$ & p.Asp116Tyr & Substitution & Missense & Severe \\
\hline Exon 4 & c. $427 \_428$ delGA & p.Glu124del & Deletion & Small deletion & Severe \\
\hline Exon 4 & c. $436 \mathrm{~A}>\mathrm{T}$ & p.Lys 127Stop & Substitution & Nonsense & Severe \\
\hline Exon 3 & c. $440 \mathrm{G}>\mathrm{A}$ & p.Ala92Thr & Substitution & Missense & Severe \\
\hline Exon 4 & c. $446 \mathrm{C}>\mathrm{T}$ & p.Pro130Leu & Substitution & Missense & Severe \\
\hline Exon 4 & c.450_51 insT & p.Gly132ins & Insertion & Insertion & Severe \\
\hline Exon 4 & c. $484 \_85$ delGA & p.Glu143del & Deletion & Small deletion & Severe \\
\hline Exon 4 & c. $518 \mathrm{~T}>\mathrm{G}$ & p.Leu154Arg & substitution & Missense & Mild \\
\hline Exon 4 & c. $523 \mathrm{~A}>\mathrm{G}$ & p.Tyr156Cys & Substitution & Missense & Moderate \\
\hline Exon 4 & c. $555 \mathrm{G}>\mathrm{A}$ & p.Asp167Lys & Substitution & Missense & Moderate \\
\hline Exon 4 & c. $575 \mathrm{~T}>\mathrm{C}$ & p.Ile173Thr & Substitution & Missense & Moderate \\
\hline Exon 4 & c. $578 \mathrm{G}>\mathrm{A}$ & p.Gly174Glu & Substitution & Missense & Severe \\
\hline Exon 4 & c. $.584 \mathrm{~T}>\mathrm{G}$ & p.Leu176Arg & Substitution & Missense & Severe \\
\hline Intron 4 & c. $602-1 \mathrm{G}>\mathrm{A}$ & & Substitution & Splicing & Severe \\
\hline Intron 4 & c. $601+5 \mathrm{G}>\mathrm{T}$ & & substitution & Splicing & Mild \\
\hline Intron 4 & c. $602-13+$ c.602_615del & & Deletion & Small deletion & Severe \\
\hline Exons $5-10$ & & & Deletion & Large deletion & Severe \\
\hline Exon 5 & c.648_49 ins10bp & p.Lys198ins & Insertion & Insertion & Severe \\
\hline Exon 5 & c. $650 \mathrm{~T}>\mathrm{C}$ & p.Lys198Pro & Substitution & Missense & Severe \\
\hline Exon 5 & c. 654 delT & p.Phe199del & Deletion & Small deletion & Severe \\
\hline Exon 5 & c.668_670 delAAG & p.Glu204del & Deletion & Small deletion & Severe \\
\hline
\end{tabular}


Table 1 continued

\begin{tabular}{|c|c|c|c|c|c|}
\hline Location & Nucleotide & Protein & Type & Effect & $\begin{array}{l}\text { Clinical } \\
\text { phenotype }\end{array}$ \\
\hline Intron 5 & c. $670+6 \mathrm{~A}>\mathrm{G}$ & & Substitution & Splicing & Mild \\
\hline Exon 6 & c.677_9delGTT & p.Ser247_Arg248delinsArg & Deletion & Small deletion & Severe \\
\hline Exon 6 & c. $686 \mathrm{C}>\mathrm{G}$ & p.Ser210Stop & Substitution & Nonsense & Severe \\
\hline Exon 6 & c. $709 \mathrm{C}>\mathrm{T}$ & p.Gln218Stop & Substitution & Nonsense & Severe \\
\hline Exon 6 & c. $741 \mathrm{G}>\mathrm{A}$ & p.Trp228Stop & Substitution & Nonsense & Severe \\
\hline Exon 6 & c. $758 \mathrm{~T}>\mathrm{G}$ & p.Val234Gly & Substitution & Missense & Severe \\
\hline Exon 6 & c.761 A > G & p.Asn235Ser & Substitution & Missense & Severe \\
\hline Exon 6 & c.763 G > T & p.Gly236Cys & Substitution & Missense & Severe \\
\hline Exon 6 & c. $776 \mathrm{G}>\mathrm{C}$ & p.Arg240Thr & Substitution & Missense & Mild \\
\hline Exon 6 & c.785 delC & p.Pro243del & Deletion & Small deletion & Moderate \\
\hline Intron 6 & c. $787+5 \mathrm{G}>\mathrm{A}$ & & Substitution & Splicing & Severe \\
\hline Exon 7 & c. $886 \mathrm{C}>\mathrm{T}$ & p.Leu277Phe & Substitution & Missense & Severe \\
\hline Exon 7 & c. 919 delA & p.Ile288del & Deletion & Small deletion & Severe \\
\hline Exon 7 & c. $967 \mathrm{G}>\mathrm{A}$ & p.Gly304Arg & Substitution & Missense & Severe \\
\hline Exon 7 & c. $980 \mathrm{~T}>\mathrm{G}$ & p.Leu308Arg & Substitution & Missense & Severe \\
\hline Exon 7 & c. $986 \mathrm{G}>\mathrm{A}$ & p.Cys310Tyr & Substitution & Missense & Severe \\
\hline Exon 8 & c. $1030 \mathrm{~A}>\mathrm{C}$ & p.Lys325Glu & Substitution & Missense & Mild \\
\hline Exon 8 & c. $1202 \mathrm{G}>\mathrm{C}$ & p.Trp382Cys & Substitution & Missense & Mild \\
\hline Exon 9 & c. $1313 \mathrm{~T}>\mathrm{C}$ & p.Ile419Tyr & Substitution & Missense & Mild \\
\hline Exon 9 & c. $1315 \mathrm{G}>\mathrm{T}$ & p.Gly420Cys & Substitution & Missense & Severe \\
\hline Exon 9 & c. $1316 \mathrm{G}>\mathrm{C}$ & p.Gly420Ala & Substitution & Missense & Moderate \\
\hline Exon 9 & c. $1322 \mathrm{~T}>\mathrm{C}$ & p.Tyr423Asn & Substitution & Missense & Severe \\
\hline Exon 9 & c. $1364 \mathrm{~T}>\mathrm{C}$ & p.Phe436Ser & Substitution & Missense & Severe \\
\hline Exon 9 & c. $1396 \mathrm{G}>\mathrm{A}$ & p.Gly447Arg & Substitution & Missense & Severe/moderate \\
\hline Exon 9 & c. $1414 \mathrm{~T}>\mathrm{A}$ & p.Leu453His & Substitution & Missense & Moderate \\
\hline Intron 9 & c. $1443+1 \mathrm{G}>\mathrm{C}$ & & Substitution & Splicing & Moderate \\
\hline Intron 9 & c. $1443+1 \mathrm{G}>\mathrm{T}$ & & Substitution & Splicing & Severe \\
\hline Exon 10 & c.1464_1465 insCCAGAC & p.Ser470ins & Insertion & Insertion & Moderate \\
\hline Exon 10 & c.1473_1474 insCCAGAC & p.Tyr473ins & Insertion & Insertion & Moderate \\
\hline Intron 10 & c. $1538-18 \mathrm{G}>\mathrm{A}$ & & Substitution & Splicing & Moderate \\
\hline Intron 10 & c. $1538-1 \mathrm{~A}>\mathrm{G}$ & & Substitution & Splicing & Severe \\
\hline Intron 10 & c. $1537+1 \mathrm{G}>\mathrm{C}$ & & Substitution & Splicing & Severe \\
\hline Exon 11 & c.1582_88 delTTCAAAT & p.Phe509_Tyr511del & Deletion & Small deletion & Severe \\
\hline Exon 11 & c. $1640 \mathrm{G}>\mathrm{A}$ & p.Cys528Tyr & Substitution & Missense & Moderate \\
\hline Exon 11 & c. $1738 \mathrm{C}>\mathrm{T}$ & p.Gln561Stop & Substitution & Nonsense & Severe \\
\hline Exon 11 & c.1748 delA & p.Asn564del & Deletion & Small deletion & Severe \\
\hline Exon 12 & c. $1810 \mathrm{~T}>\mathrm{C}$ & p.Trp585Arg & Substitution & Missense & Severe \\
\hline Exon 12 & c. $1811 \mathrm{G}>\mathrm{A}$ & p.Trp585Stop & Substitution & Nonsense & Severe \\
\hline Exon 12 & c. $1813 \mathrm{~T}>\mathrm{C}$ & p.Tyr586His & Substitution & Missense & Severe \\
\hline Exon 12 & c. $1814 \mathrm{~A}>\mathrm{G}$ & p.Tyr586Cys & Substitution & Missense & Severe \\
\hline Exon 12 & c. 1845 delC & p.Pro596del & Deletion & Small deletion & Severe \\
\hline Exon 12 & c.1874_75 delCA & p.Pro606 del & Deletion & Small deletion & Severe \\
\hline Exon 12 & c. 1897 A > G & p.Met614Val & Substitution & Missense & Mild \\
\hline Intron 12 & c. $1904-2 \mathrm{~A}>\mathrm{G}$ & & Substitution & Splicing & Severe \\
\hline Intron 12 & c. $1904-1 \mathrm{G}>\mathrm{A}$ & & Substitution & Splicing & Severe \\
\hline Intron 12 & c. $1903+2 \mathrm{~T}>\mathrm{G}$ & & Substitution & Splicing & Severe \\
\hline Exon 12 & c. $1909 \mathrm{G}>\mathrm{T}$ & p. Glu581Asp & Substitution & Missense & Severe \\
\hline
\end{tabular}


Table 1 continued

\begin{tabular}{|c|c|c|c|c|c|}
\hline Location & Nucleotide & Protein & Type & Effect & $\begin{array}{l}\text { Clinical } \\
\text { phenotype }\end{array}$ \\
\hline Exon 13 & c. $1913 \mathrm{G}>\mathrm{A}$ & p.Gly619Asp & Substitution & Missense & Severe \\
\hline Exon 13 & c. $1945 \mathrm{~T}>\mathrm{C}$ & p.Cys630Arg & Substitution & Missense & Severe \\
\hline Exon 13 & c. $1966 \mathrm{~T}>\mathrm{C}$ & p.Trp637Arg & Substitution & Missense & Severe \\
\hline Exon 13 & c. $1966 \mathrm{~T}>\mathrm{G}$ & p.Trp637Gly & Substitution & Missense & Moderate \\
\hline Exon 13 & c. $1991 \mathrm{~A}>\mathrm{G}$ & p.Gln645Arg & Substitution & Missense & Moderate \\
\hline Exon 13 & c. $2029 \mathrm{~T}>\mathrm{G}$ & p.Phe658Val & Substitution & Missense & Moderate \\
\hline Exon 13 & c.2058_2059 delAC & p.Thr668del & Deletion & Small deletion & Severe \\
\hline Exon 13 & c. $2061 \mathrm{C}>\mathrm{T}$ & p.Pro672Ser & Substitution & Missense & Severe \\
\hline Exon 13 & c. $2078 \mathrm{C}>\mathrm{G}$ & p.Ser674Stop & Substitution & Nonsense & Severe \\
\hline Exon 13 & c. $2081 \mathrm{G}>\mathrm{A}$ & p.Gly675Glu & Substitution & Missense & Severe/moderate \\
\hline Exon 13 & c. $2090 \mathrm{~T}>\mathrm{C}$ & p.Val678Ala & Substitution & Missense & Mild \\
\hline Exon 13 & c. $2102 \mathrm{~T}>\mathrm{C}$ & p.Met682Thr & Substitution & Missense & Mild \\
\hline Exons 14-26 & & & Deletion & Large deletion & Severe \\
\hline Exon 14 & c. $2161 \mathrm{~A}>\mathrm{G}$ & p.Met702Val & Substitution & Missense & Severe \\
\hline Exon 14 & c. 2202 delT & p.Thr715del & Deletion & Small deletion & Severe \\
\hline Exon 13 & c.2032_37 delAAACAC & p.Lys659_His660del & Deletion & Small deletion & Severe \\
\hline Exon 14 & c. 2216 delA & p.Glu720del & Deletion & Small deletion & Severe \\
\hline Exon 14 & $\begin{array}{l}\text { c. } 2521 \_30 \\
\text { delCCTGGAGCAA }\end{array}$ & p.Pro822_Ile825del & Deletion & Small deletion & Severe \\
\hline Exon 14 & c.2534_37 delACAG & p.Asp826_Ser827del & Deletion & Small deletion & Severe \\
\hline Exon 14 & c.2729_30 insC & p.Ser891ins & Insertion & Insertion & Severe \\
\hline Exon 14 & c. $2742 \mathrm{~T}>\mathrm{G}$ & p.Leu859Stop & Substitution & Nonsense & Severe \\
\hline Exon 14 & c. $2777 \_78$ insC & p.Pro907ins & Insertion & Insertion & Severe \\
\hline Exon 14 & c. 2810 delA & p.Asp918del & Deletion & Small deletion & Severe \\
\hline Exon 14 & c. $3113 \mathrm{C}>\mathrm{G}$ & p.Ser1019Stop & Substitution & Nonsense & Severe \\
\hline Exon 14 & c. 3224 delC & p.Ala1056del & Deletion & Small deletion & Severe \\
\hline Exon 14 & c. $3381 \mathrm{G}>\mathrm{A}$ & p.Trp1108Stop & Substitution & Nonsense & Severe \\
\hline Exon 14 & c. 3430 delA & p.Ser1125del & Deletion & Small deletion & Severe \\
\hline Exon 14 & c.3619_20 delCA & p.His1188del & Deletion & Small deletion & Severe \\
\hline Exon 14 & c.3625_26 insC & p.Asn1189ins & Insertion & Insertion & Severe \\
\hline Exon 14 & c.3652 delG & p.Glu1199del & Deletion & Small deletion & Severe \\
\hline Exon 14 & c.3834 delA & p.Arg1260del & Deletion & Small deletion & Severe \\
\hline Exon 14 & c. $3865 \mathrm{~A}>\mathrm{T}$ & p.Lys1270Stop & Substitution & Nonsense & Severe \\
\hline Exon 14 & c.3904 delCAAAC & p.Gln1282_Asn1283del & Deletion & Small deletion & Severe \\
\hline Exon 14 & c.3911_12 insA & p.Lys1285ins & Insertion & Insertion & Severe \\
\hline Exon 14 & c.3984_85 insA & p.Arg1310ins & Insertion & Insertion & Severe \\
\hline Exon 14 & c. $4067 \mathrm{C}>\mathrm{A}$ & p.Ser1337Stop & Substitution & Nonsense & Severe \\
\hline Exon 14 & c. $4077 \mathrm{G}>\mathrm{A}$ & p.Trp1340Stop & Substitution & Nonsense & Severe \\
\hline Exon 14 & c.4097 delT & p.Lys 1347 del & Deletion & Small deletion & Severe \\
\hline Exon 14 & c. $4114 \mathrm{~A}>\mathrm{G}$ & p.Thr1353Ala & Substitution & Missense & Severe \\
\hline Exon 14 & c. $4156 \mathrm{C}>\mathrm{T}$ & p.Gln1367Stop & Substitution & Nonsense & Severe \\
\hline Exon 14 & c. $4363 \mathrm{C}>\mathrm{T}$ & p.Gln1436Stop & Substitution & Nonsense & Severe \\
\hline Exon 14 & c.4388_91 delCTTT & p.Ser1444_Leu1445del & Deletion & Small deletion & Severe \\
\hline Exon 14 & c.4477_80 delAAAG & p.Lys1474_Val1475del & Deletion & Small deletion & Severe \\
\hline Exon 14 & c. 4483 delG & p.Glu1476del & Deletion & Small deletion & Severe \\
\hline Exon 14 & c. $4483 \mathrm{G}>\mathrm{T}$ & p.Glu1476Stop & Substitution & Nonsense & Severe \\
\hline Exon 14 & c. 4687 delG & p.Val1544del & Deletion & Small deletion & Severe \\
\hline Exon 14 & c. $4774 \_75$ insA & p.Thr1573ins & Insertion & Insertion & Severe \\
\hline
\end{tabular}


Table 1 continued

\begin{tabular}{|c|c|c|c|c|c|}
\hline Location & Nucleotide & Protein & Type & Effect & $\begin{array}{l}\text { Clinical } \\
\text { phenotype }\end{array}$ \\
\hline Exon 14 & c.4788_89 insA & p.Glu1578ins & Insertion & Insertion & Severe \\
\hline Exon 14 & c.4825_26 insA & p.Thr1590ins & Insertion & Insertion & Severe \\
\hline Exon 14 & c.4895_96 insT & p.Ile1613ins & Insertion & Insertion & Severe \\
\hline Exon 14 & c.4921_22 insT & p.Ile1622ins & Insertion & Insertion & Severe/moderate \\
\hline Exon 14 & c. $5063 \mathrm{C}>\mathrm{T}$ & p.Ser1669Leu & Substitution & Missense & Moderate \\
\hline Exon 14 & c. 5125 delA & p.Ser1690del & Deletion & Small deletion & Severe \\
\hline Exon 14 & c. $5176 \mathrm{~T}>\mathrm{C}$ & p.Trp1707Arg & Substitution & Missense & Severe \\
\hline Exon 14 & c. $5184 \mathrm{~T}>\mathrm{A}$ & p.Tyr1709Stop & Substitution & Nonsense & Severe \\
\hline Exon 14 & c.5213_16 delGAAA & p.Arg1719_Asn1720del & Deletion & Small deletion & Severe \\
\hline Intron 14 & c. $5219+1 \mathrm{G}>\mathrm{A}$ & & Substitution & Splicing & Severe \\
\hline Intron 14 & c. $5219+2 \mathrm{~T}>\mathrm{C}$ & & Substitution & Splicing & Severe \\
\hline Intron 14 & c. $5220-2 \mathrm{~A}>\mathrm{T}$ & & Substitution & Splicing & Severe \\
\hline Exon 15 & del 3bp inser22bp & p.Val1727delins22bp & Complex & $\begin{array}{l}\text { Insertion/small } \\
\text { deletion }\end{array}$ & Severe \\
\hline Exon 15 & c. $5253 \mathrm{~A}>\mathrm{T}$ & p.Lys1732Asn & Substitution & Missense & Severe \\
\hline Exon 15 & c. $5275 \mathrm{G}>\mathrm{A}$ & p.Asp1740Asn & Substitution & Missense & Mild \\
\hline Exon 15 & c. $5321 \mathrm{~A}>\mathrm{G}$ & p.His1755Arg & Substitution & Missense & Severe \\
\hline Intron 16 & c. $5373+1 \mathrm{G}>\mathrm{A}$ & & Substitution & Splicing & Severe \\
\hline Intron 16 & c. $5373+2 \mathrm{~T}>\mathrm{G}$ & & Substitution & Splicing & Moderate \\
\hline Exon 16 & c. $5387 \mathrm{~A}>\mathrm{G}$ & p.Asn1777 Ser & Substitution & Missense & Severe \\
\hline Exon 16 & c. $5398 \mathrm{C}>\mathrm{A}$ & p.Arg1781Ser & Substitution & Missense & Severe \\
\hline Exon 16 & c. $5398 \mathrm{C}>\mathrm{T}$ & p.Arg1781Cys & Substitution & Missense & Moderate \\
\hline Exon 16 & c.5405 A > C & p.Tyr1783Arg & Substitution & Missense & Severe \\
\hline Exon 16 & c. $5413 \mathrm{~T}>\mathrm{A}$ & p.Tyr1786Asn & Substitution & Missense & Severe \\
\hline Exon 16 & c. $5419 \mathrm{~A}>\mathrm{G}$ & p.Ser1788Gly & Substitution & Missense & Mild \\
\hline Exon 16 & c.5473_5478 delTTTGTC & p.Phe1806_Val1807del & Deletion & Small deletion & Severe \\
\hline Exon 14 & c.5501_5502 insA & p.Trp1815ins & Insertion & Insertion & Severe \\
\hline Exon 16 & c. $5515 \mathrm{C}>\mathrm{T}$ & p.Gln1820Stop & Substitution & Nonsense & Severe \\
\hline Exon 16 & c. $5522 \mathrm{~A}>\mathrm{T}$ & p.His1822Leu & Substitution & Missense & Mild \\
\hline Exon 16 & c. $5561 \mathrm{~T}>\mathrm{G}$ & p.Trp1835Gly & Substitution & Missense & Severe \\
\hline Exon 17 & c. $5662 \mathrm{~A}>\mathrm{T}$ & p.Arg1869Stop & Substitution & Nonsense & Severe \\
\hline Exon 17 & c. $5674 \mathrm{G}>\mathrm{A}$ & p.Val1873Ile & Substitution & Missense & Moderate \\
\hline Exon 17 & c.5688_89 delCT & p.Leu1878del & Deletion & Small deletion & Moderate \\
\hline Exon 17 & c. $5715 \mathrm{G}>\mathrm{A}$ & p.Gly1850Asp & Substitution & Missense & Severe \\
\hline Exon 18 & c. $5824 \mathrm{G}>\mathrm{A}$ & p.Gly1923Asp & substitution & Missense & Severe \\
\hline Exon 18 & c. $5837 \mathrm{~A}>\mathrm{T}$ & p.Asp1927Val & substitution & Missense & Mild \\
\hline Exon 18 & c. $5918 \mathrm{~A}>\mathrm{G}$ & p.His 1954 Arg & Substitution & Missense & Severe \\
\hline Exon 18 & c. $5998 \mathrm{G}>\mathrm{A}$ & p.Gly1981Ser & Substitution & Missense & Severe \\
\hline Exon 19 & c. $6043 \mathrm{~T}>\mathrm{C}$ & p.Trp1996Arg & Substitution & Missense & Severe \\
\hline Exon 19 & c. $6104 \mathrm{~T}>\mathrm{G}$ & p.Val2016Gly & Substitution & Missense & Severe \\
\hline Intron 19 & c. $6115+4 \mathrm{~A}>\mathrm{G}$ & & Substitution & Splicing & Severe \\
\hline Intron 19 & c. 6115 delGTG $+1 \_+3$ & & Substitution & Splicing & Severe \\
\hline Exon 19 & c. $6151 \mathrm{~A}>\mathrm{T}$ & p.Ile2032Phe & Substitution & Missense & Mild \\
\hline Intron 21 & c. $6187-1 \mathrm{G}>\mathrm{A}$ & & Substitution & Splicing & Severe \\
\hline Exon 21 & c. $6267 \mathrm{G}>\mathrm{T}$ & p.Trp2070Cys & Substitution & Missense & Mild \\
\hline Exon 22 & c. $6310 \mathrm{~A}>\mathrm{T}$ & p.Lys2085Stop & Substitution & Nonsense & Severe \\
\hline Exon 22 & c.6312_13 insG & p.Thr2086ins & Insertion & Insertion & Severe \\
\hline Exon 22 & c. $6320 \mathrm{G}>\mathrm{A}$ & p.Gly2088Asp & Substitution & Missense & Severe \\
\hline
\end{tabular}


Table 1 continued

\begin{tabular}{|c|c|c|c|c|c|}
\hline Location & Nucleotide & Protein & Type & Effect & $\begin{array}{l}\text { Clinical } \\
\text { phenotype }\end{array}$ \\
\hline Exon 21 & c. $6335 \mathrm{G}>\mathrm{T}$ & p.Gly2057Stop & Substitution & Nonsense & Severe \\
\hline Exon 22 & c. $6374 \mathrm{G}>\mathrm{C}$ & p.Ser2106Thr & Substitution & Missense & Severe \\
\hline Intron 22 & $\begin{array}{l}\text { c. } 6430-21+ \\
\quad \text { c. } 6430 \_6439 \text { del }\end{array}$ & & Deletion & Small deletion & Severe \\
\hline Exon 23 & c. $6506 \mathrm{G}>\mathrm{T}$ & p.Arg2150Leu & Substitution & Missense & Mild \\
\hline Exon 23 & c. $6546 \mathrm{~T}>\mathrm{C}$ & p.Phe2127Ser & Substitution & Missense & Mild \\
\hline Exon 23 & c. $6560 \mathrm{G}>\mathrm{A}$ & p.Gly2168Asp & Substitution & Missense & Severe \\
\hline Exon 23 & c. $6563 \mathrm{G}>\mathrm{A}$ & p.Cys2169Tyr & Substitution & Missense & Moderate \\
\hline Exon 24 & c. $6593 \mathrm{G}>\mathrm{A}$ & p.Gly2179Glu & Substitution & Missense & Severe \\
\hline Exon 24 & c. $6593 \mathrm{G}>\mathrm{T}$ & p.Gly2179Val & Substitution & Missense & Severe \\
\hline Exon 24 & $\begin{array}{l}\text { c. } 6623 \_25 \text { delAGA } \\
\text { Gln2189+ } \\
\text { c. } 6628 \text { delA Thr2191 }\end{array}$ & p.Gln2189del + p.Thr2191del & Complex & Small deletions & Severe \\
\hline Exon 24 & c. $6637 \mathrm{~T}>\mathrm{C}$ & p.Ser2194Pro & Substitution & Missense & Severe \\
\hline Exon 24 & c. $6638 \mathrm{C}>\mathrm{G}$ & p.Ser2194Cys & Substitution & Missense & Moderate \\
\hline Exon 24 & c. 6650 delA & p.Asn2198del & Deletion & Small deletion & Severe \\
\hline Exon 24 & c. $6652 \mathrm{~A}>\mathrm{G}$ & p.Met2199Val & Substitution & Missense & Severe \\
\hline Exon 25 & c. $6738 \mathrm{G}>\mathrm{A}$ & p.Glu2228Lys & Substitution & Missense & Moderate \\
\hline Exon 25 & c. $6739 \mathrm{G}>\mathrm{T}$ & p.Glu2228Stop & Substitution & Nonsense & Severe/moderate \\
\hline Exon 25 & c. $6742 \mathrm{~T}>\mathrm{C}$ & p.Trp2229Arg & Substitution & Missense & Mild \\
\hline Exon 25 & c. $6746 \mathrm{~T}>\mathrm{C}$ & p.Leu2230Pro & Substitution & Missense & Severe \\
\hline Exon 25 & c. $6776 \mathrm{~T}>\mathrm{C}$ & p.Val2240Asp & Substitution & Missense & Severe \\
\hline Exon 25 & c. $6785 \mathrm{~T}>\mathrm{C}$ & p.Val2243Ala & Substitution & Missense & Moderate \\
\hline Exon 25 & c.6894_95 insAAC & p.Val1280ins & Insertion & Insertion & Severe \\
\hline Exon 26 & c. $6907 \mathrm{C}>\mathrm{T}$ & p.Gln2284Stop & Substitution & Nonsense & Severe \\
\hline Exon 26 & c. $6956 \mathrm{C}>\mathrm{G}$ & p.Pro2300Arg & Substitution & Missense & Severe \\
\hline Exon 26 & c.6966_67 insGACT & p.Thr2303ins & Insertion & Insertion & Severe \\
\hline Exon 26 & c. $6982 \mathrm{C}>\mathrm{G}$ & p.His2309Asp & Substitution & Missense & Severe \\
\hline Exon 26 & c. $6995 \mathrm{G}>\mathrm{C}$ & p.Trp2313Ser & Substitution & Missense & Moderate \\
\hline Exon 26 & c.7015 A > T & p.Arg2320Trp & Substitution & Missense & Severe \\
\hline Exon 26 & c. $* 1 \mathrm{~T}>\mathrm{C}$ & & Substitution & Missense & Mild \\
\hline
\end{tabular}

A. Iorio; Pescara: Dr. A. Dragani; Piacenza: Dr.ssa MC. Arbasi; Ravenna: Dr. A. Mancino; Reggio Calabria: Dr. V. Trapani Lombardo; Reggio Emilia: Dr. M. D’Incà; Roma "Ospedale Pediatrico Bambin Gesù": Prof. G. De Rossi; Roma "Policlinico Universitario A. Gemelli": Prof. R. Landolfi; Roma "Policlinico Universitario La Sapienza": Prof.ssa G. Mazzucconi; Sassari: Dr. G. Piseddu; Torino "Policlinico Pediatrico": Dr.ssa L. Perugini: Dr.ssa M Messina; Torino "Le Molinette": Dr. G. Tamponi: Dr.ssa P. Schinco; Trento: Dr.ssa G. Rossetti; Udine: Dr. G. Barillari; Vallo della Lucania (SA): Dr. A. Catalano, Dr. G. Feola; Verona: Dr. G. Gandini; Vicenza: Dr. G. Castaman, S. Giacomelli, R. Ghiotto.

\section{References}

Acquila M, Pasino M, Lanza T, Bottini F, Molinari AC, Bicocchi MP (2004) Duplication of exon 13 causes one third of the cases of mild hemophilia A in northern Italy. Haematologica 89:758-759

Bagnall RD, Waseem N, Green PM, Gianelli F (2002) Recurrent inversion breaking intron 1 of the factor VIII gene is a frequent cause of severe hemophilia A. Blood 99:168-174

D’Andrea G, Colaizzo D, Vecchione G, Grandone E, Di Minno G, Margaglione M (2002) GLAnzmann's Thrombasthenia Italian Team (GLATIT). Glanzmann's thrombasthenia: identification of 19 new mutations in 30 patients. Thromb Haemost 87:10341042

Deszo D, Ventura C, Moreira I, DinizMJ, Antunes M, Tavares A, Araùjo $\mathrm{F}$, MoraisS, Campos M, Lavina $\mathrm{J}$, Kemball-Cook J (2006) The spectrum of mutations and molecular pathogenesis of 
hemophilia A in 181 Portuguese patients. Haematologica 91:840-843

Fernández-López O, García-Lozano J-R, Núñez-Vázquez R, PérezGarrido R, Núñez-Roldán A (2005) The spectrum of mutations in Southern Spanish patients with hemophilia A and identification of 28 novel mutations. Haematologica 90:707-710

Gitschier J, Wood WI, Goralka TM, Wion KL, Chen EY, Eaton DH, Vehar GA, Capon DJ, Lawn RM (1984) Characterization of the human factor VIII gene. Nature 312:326-330

Habart D, Kalakova D, Novotay M, Vorlova Z (2003) Thirty-four novel mutations detected in factor VIII gene by multiplex CSGE: modeling of 13 novel amino acid substitutions. J Thromb Haemost 1:773-778

Jayandharan G, Shaji RV, Baidya S, Nair SC, Chandy M, Srivastava A (2005) Identification of factor VIII gene mutations in 101 patients with haemophilia A: mutation analysis by inversion screening and multiplex PCR and CSGE and molecular modelling of 10 novel missense substitutions. Haemophilia 11:481491

Kazazian HH, Tuddenham EGD, Antonarakis SE (1995) Hemophilia A and parahemophilia: deficiencies of coagulation factors VIII and V. In: Scriver CR, Beaudet AL, Sly WS, Valle D (eds) The metabolic and molecular bases of inherited disease, 7th edn. McGraw-Hill, New York, pp 3241-3267

Kemball-Cook G, Tuddenham EG, Wacey AI (1998) The factor VIII structure and mutation resource site: HAMSTeRS version 4. Nucleic Acids Res 26:216-219

Liu Q, Nozari G, Sommer SS (1998) Single-tube polymerase chain reaction for rapid diagnosis of the inversion hotspot of mutation in hemophilia A. Blood 92:1458-1459

Ljung RC, Sjorin E (1999) Origin of mutation in sporadic cases of hemophilia A. Br J Haematol 106:870-874

Miller SA, Dykes DD, Polesky HF (1988) A simple salting out procedure for extracting DNA from human nucleated cells. Nucleic Acids Res 16:1215

Oldenburg J, Pavlova A (2006) Genetic risk factors for inhibitors to factor VIII and IX. Haemophilia 12(Suppl 6):15-22

White GC 2nd, Rosendaal F, Aledort LM, Lusher JM, Rothschild C, Ingerslev J, Factor VIII, Factor IX Subcommittee (2001) Definitions in hemophilia. Recommendation of the scientific subcommittee on factor VIII and factor IX of the scientific and standardization committee of the International Society on Thrombosis and Haemostasis. Thromb Haemost 85:560 